\title{
Mechanical positive pressure ventilation during resuscitation in out-of-hospital cardiac arrest with Chest Compression Synchronized Ventilation (CCSV)
}

Clemens Kill ${ }^{1}$, Christian Paul2 ${ }^{2}$, Randi Manegold ${ }^{1}$, Carola Holzner ${ }^{1}$, Ralf Blomeyer ${ }^{2}$, Joachim Risse ${ }^{1}$ ${ }^{1}$ Center of Emergency Medicine, University Hospital Essen, Germany; ${ }^{2}$ Cologne Fire Departement, Cologne, Germany

\section{Purpose of the study}

The ILCOR-Guidelines recommend ventilation with pure oxygen and Intermitted Positive Pressure Ventilation with a fixed respiration rate of 10/min during Advanced Life Support (ALS)[1]. Meanwhile there is a novel resuscitation ventilation mode named Chest Compression Synchronized Ventilation (CCSV) available[2]. We report data of first cases of resuscitation with CCSV in out-ofhospital cardiac arrest (OHCA).

\section{Materials and methods}

In an urban physician staffed Emergency Medical Service ventilation with CCSV following endotracheal intubation was added to standard ALS-algorithms. CCSV is designed as a pressure-controlled and with each chest compression synchronized breathing pattern $\left(\mathrm{FiO}_{2} 1.0\right.$, Pinsp $=60 \mathrm{mbar}$, inspiratory time $205 \mathrm{~ms}$ ), insufflating a very short oxygen flow in time with the start of each chest compression. We retrospectively evaluated all cases of OHCA with use of CCSV and measured time of mechanical ventilation with both manual and mechanical chest compression (CC) (median, $25 \% / 75 \%$ percentiles), number of patients admitted to hospital with return of spontaneous circulation (ROSC), 24h-survival and discharged alive.

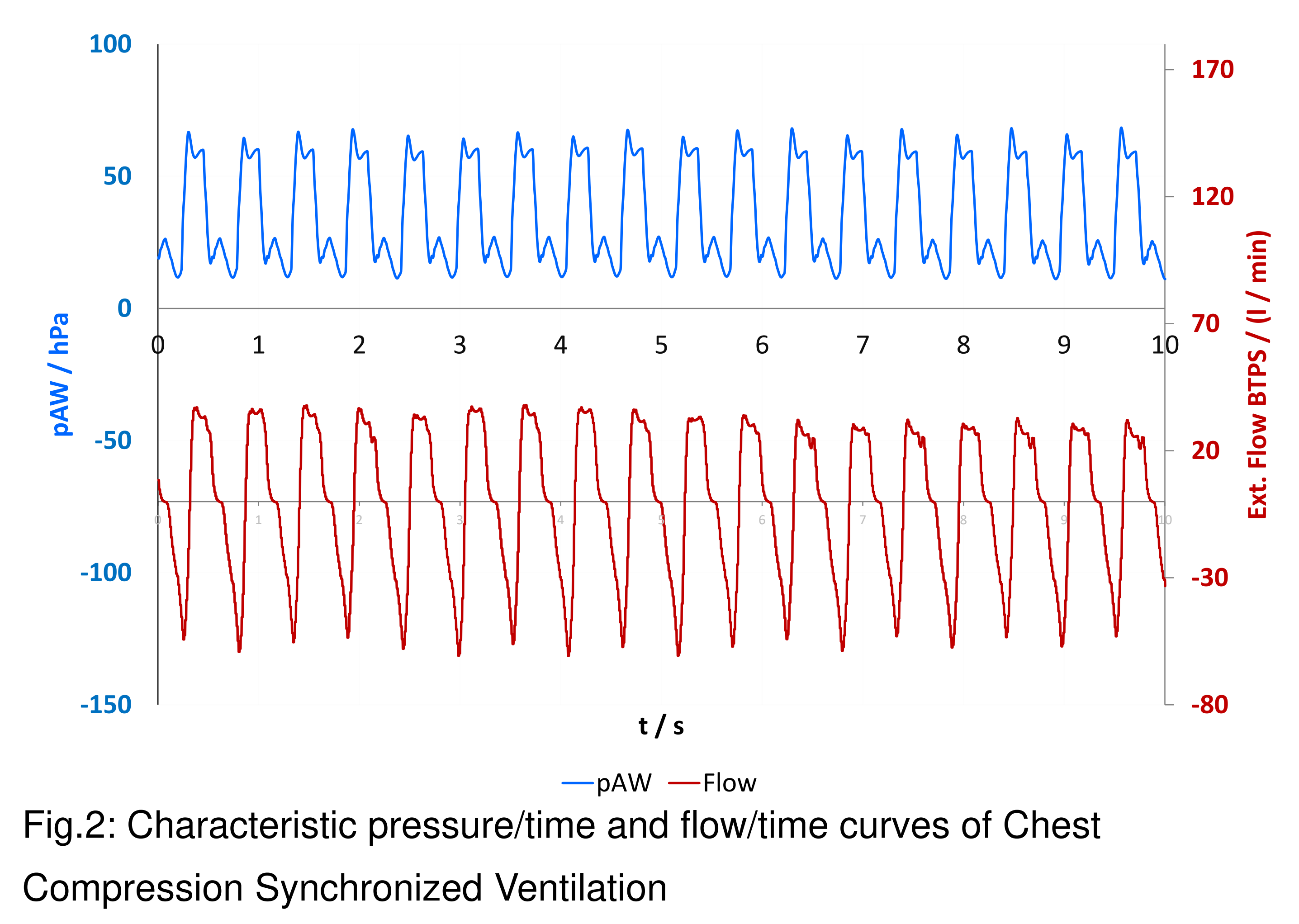

Fig.1: Chest Compression Synchronized Ventilation in out-ofhospital cardiac arrest on scene

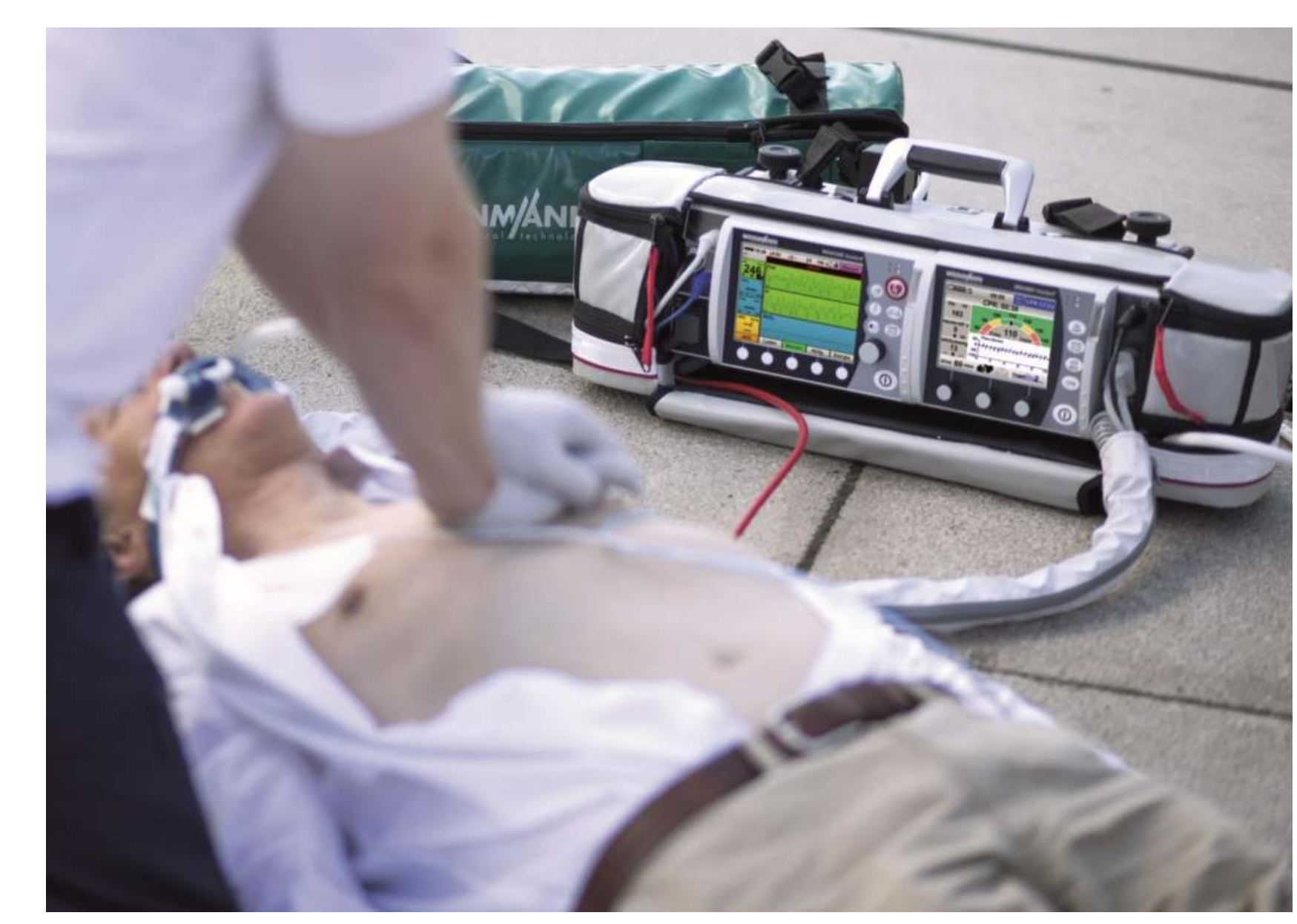

\section{Results}

34 Patients with OHCA were ventilated with CCSV between July 2018 and March 2019. CCSV Ventilation time in total $(n=34)$ 18:25(7:54/26:17)min; manual CC $(n=16)$ 5:04(2:04/6:38)min, mechanical CC $(n=29)$ 18:26(7:23/26:42)min. Admission with ROSC $\mathrm{n}=21(61.8 \%), \quad 24 \mathrm{~h}$ survival $\mathrm{n}=12(35.3 \%)$, discharged alive $n=5(14.7 \%)$. Total CCSVVentilation times in patients discharged alive were $4: 48,8: 42,25: 00,30: 18$ and 40:24min. No serious adverse events by CCSV were reported.

\section{Conclusions}

This is the first reported case series of Chest Compression Synchronized Ventilation (CCSV) in OHCA. The rates of ROSC in admission and outcome are promising high with a maximum time of ventilation during chest compression of more than 40 minutes. Therefore we need further data to evaluate the potential of CCSV.

\section{References}

[1]Soar J: Resuscitation 2015(95):100-147

[2]Kill C: Crit Care Med. 2014(42):e89-95

\author{
Contact \\ Center of Emergency Medicine, \\ University Hospital Essen \\ Hufelandstr. 55 \\ D-45147 Essen \\ Email: Clemens.Kill@UK-Essen.de
}

